\title{
Four-Wave-Mixing Enhancement in Optical Microfibers
}

\author{
Muhammad I.M. Abdul Khudus ${ }^{1,2}$, Francesco De Lucia ${ }^{1}$, Costantino Corbari ${ }^{1}$, Timothy Lee ${ }^{1}$, \\ Pier Sazio $^{1}$, Peter Horak ${ }^{1}$ and Gilberto Brambilla ${ }^{1}$ \\ ${ }^{1}$ Optoelectronics Research Centre, University of Southampton, Southampton, SO17 1BJ, United Kingdom \\ ${ }^{2}$ Photonics Research Centre, Department of Physics, Faculty of Science, University of Malaya, 50603 Kuala Lumpur, Malaysia \\ Author e-mail address: miak2g12@soton.ac.uk
}

\begin{abstract}
Four-Wave-Mixing (FWM) is enhanced by tailoring the modal effective index in optical microfibers to improve the phase matching conditions. An enhancement of $>25 \mathrm{~dB}$ was recorded in a 6-mm-long optical microfiber.
\end{abstract}

OCIS codes: (190.0190) Nonlinear optics; (060.2310) Fiber optics; (060.4370) Nonlinear optics, fibers;

\section{Introduction}

Four wave mixing (FWM) is a well-known nonlinear process which occurs when two incoming photons from either one or two signals are annihilated, creating two photons at two different frequencies of light [1]. This process allows for the conversion or amplification of coherent signals and has been demonstrated in a wide number of waveguides including optical fibers, microstructured fibers and dispersion shifted fibers [2-6]. As the process exploits the Kerr effect in a medium, it is more easily achieved in a medium with relatively high nonlinearity [7].

Optical microfibers (OMF) are a prime candidate for such an application, as they have the advantage of having a relatively high nonlinearity, good confinement and a tailorable dispersion. Indeed, it has been shown that it is possible to produce cascaded FWM spanning almost an octave in frequency by employing relatively short OMFs [8]. Here, we show that tailoring the OMF diameter and changing its dispersion allows for better phase matching, thereby amplifying the FWM signal by at least two orders of magnitude.

\section{Experimental Setup}

In general, for pump signals at frequencies $\omega_{1}$ and $\omega_{2}$, efficient FWM producing a signal at $\omega_{3}$ and an idler at $\omega_{4}$ requires the conservation of energy and momentum, which results in the two basic criteria, [6]:

$$
\begin{gathered}
\Delta \beta=\beta\left(\omega_{4}\right)+\beta\left(\omega_{3}\right)-\beta\left(\omega_{1}\right)-\beta\left(\omega_{2}\right)=0 \\
\omega_{1}+\omega_{2}=\omega_{3}+\omega_{4}
\end{gathered}
$$

where $\beta(\omega)$ is the propagation constant of a particular mode at the frequency $\omega$. Here, degenerate FWM, where $\omega_{1}=\omega_{2}$, with only the fundamental mode at all three wavelengths is investigated.

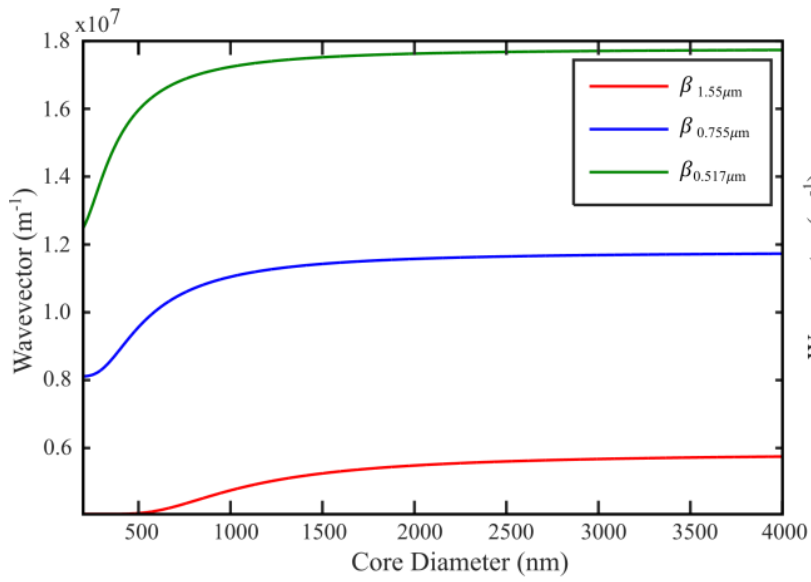

(a)

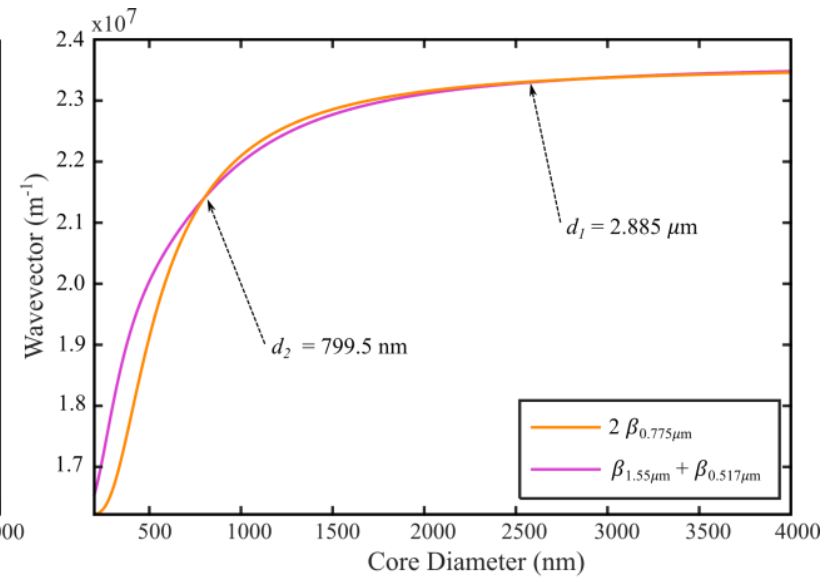

(b)

Figure 1: (a) Dependence of the wavevector $\beta_{\lambda}$ on the OMF diameter at the wavelengths $\lambda_{\text {signal }}=1.55 \mu \mathrm{m}, \lambda_{\text {pump }}=0.775 \mu \mathrm{m}$ and $\lambda_{\text {idler }}=$ $0.517 \mu \mathrm{m}$. (b) Phase matching requirement (eq. 1) for degenerate FWM. The intersection points indicate the phase matching diameter for efficient FWM.

To find the phase matching diameters which satisfy (1), the rigorous eigenvalue equations for a step index profile was solved. Only silica core OMFs were considered for degenerate FWM with a pump at $775 \mathrm{~nm}$, signal at 
$1550 \mathrm{~nm}$ and idler at $516.7 \mathrm{~nm}$. The wavevector variation of the fundamental modes at the three wavelengths is shown in figure 1 (a). Phase matching requires $2 \beta\left(\omega_{775 \mathrm{~nm}}\right)=\beta\left(\omega_{1550 \mathrm{~nm}}\right)+\beta\left(\omega_{516.7 \mathrm{~nm}}\right)$, and it is represented by intersection of the two curves in Fig. 1(b). Two phase matching diameters can be clearly distinguished, namely, at $d_{1}=2.885 \mu \mathrm{m}$ and $d_{2}=799.5 \mathrm{~nm}$. This procedure is similar to the procedure to find the phase matching diameter for third harmonic generation in OMF [9].

In order to investigate the possibility of using OMF for enhancing FWM, a Master Oscillator Parametric Amplifier (MOPA) with a tunable seed laser working in the C-band (1530-1565 nm) is employed, as shown in Fig. 2. The MOPA produces $5 \mathrm{~ns}$ pulses with a repetition rate of $200 \mathrm{kHz}$, average powers of between $300 \mathrm{~mW}$ and 800 $\mathrm{mW}$, and peak powers of approximately $300 \mathrm{~W}$ to $800 \mathrm{~W}$. The output of the MOPA is then passed through a polarization controller (PC) and a periodically poled silica fiber (PPSF). This partially converts the MOPA signal into a signal at the second harmonic wavelength at about $775 \mathrm{~nm}$. To avoid pump depletion and other nonlinear effects, an average power of only $300 \mathrm{~mW}$ was used at $1550 \mathrm{~nm}$, resulting in a signal of more than $3 \mathrm{~mW}$ at $775 \mathrm{~nm}$. The output is connected to a pure silica core Z-Fiber (Sumitomo Electric) which is then tapered using the modified flame brushing method [7]. The resulting signal is spliced onto a bend shortpass filter before being connected to a broadband OSA (Yokogawa AQ6315A) operating between $350 \mathrm{~nm}$ and $1700 \mathrm{~nm}$.

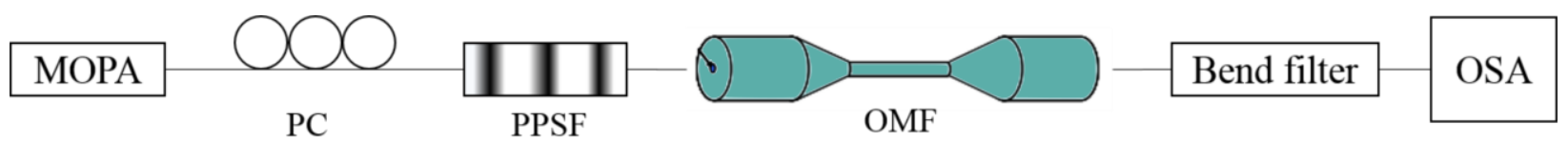

Figure 2: Experimental setup used to amplify FWM in OMF. The output signal from the PPSF is launched into the OMF being tapered. To avoid pump depletion, the MOPA output was kept at an average power of $300 \mathrm{~mW}$. The bend filter is used to remove the pump and signal wavelengths.

The output of the OMF at the idler wavelength $(\lambda=0.517 \mu \mathrm{m})$ is continuously monitored whilst tapering, with the predicted diameter of the process recorded, thereby allowing any change in the FWM signal to be recorded.

\section{Results and Discussion}

The interaction between the fundamental and second harmonic at the output of the PPSF allows for the observation of FWM at $\lambda=0.517 \mu \mathrm{m}$, satisfying the condition imposed on the frequency difference in (2). This is due to the fact that both signals have relatively high peak powers, that they are automatically co-polarized and would spatially overlap as a result of the second harmonic generation process. The use of ns pulses reduces the possibility of walkoff, increasing the interaction length for FWM. The initial output signal after the bend filter before any tapering is done is shown in figure 3 .

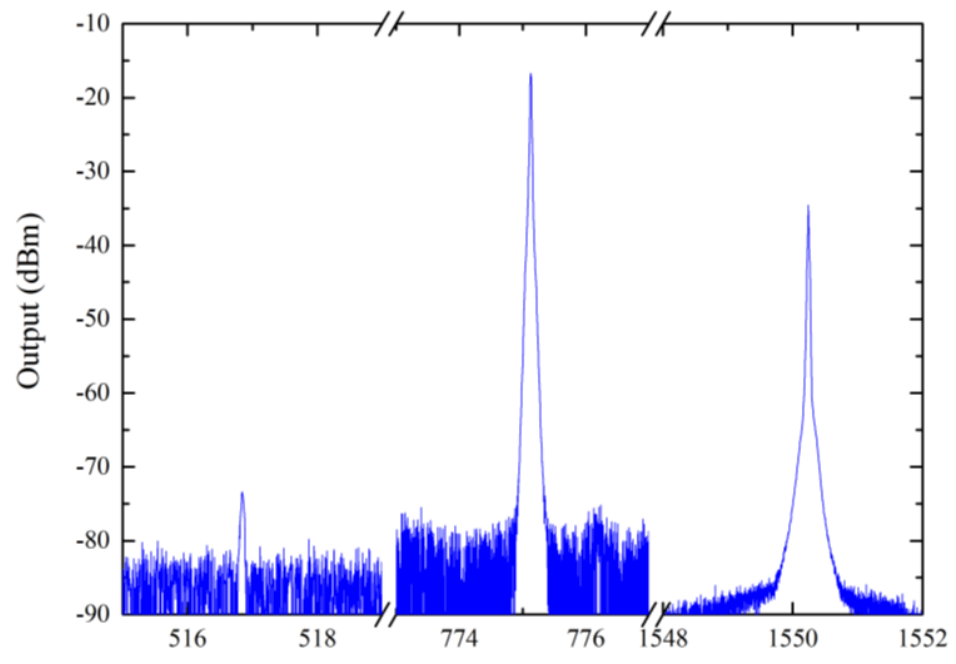

Wavelength (nm)

Figure 3: The initial output signal before the Z-Fiber is tapered. The loss of the bend filter is wavelength dependent, with higher losses at longer wavelengths, therefore causing the signal at $1550 \mathrm{~nm}$ to appear smaller. The initial FWM signal at $516.7 \mathrm{~nm}$ is very small and is just above the noise level. This signal is increased significantly at the phase matching diameters, as shown in figure 4.

A section of the Z-Fiber is then tapered whilst the MOPA is turned on, and the change in output of the FWM centered at the peak of the idler signal with diameter recorded as shown in figure 4 . The signal is measured with a 
resolution of $10 \mathrm{~nm}$ allowing for the entire signal to be captured. The OMF is tapered to a diameter of $0.5 \mu \mathrm{m}$ from an initial diameter of $125 \mu \mathrm{m}$, with the tapering profile controlled carefully to satisfy the adiabaticity criteria in order to minimize loss [7]. The final diameter of $0.5 \mu \mathrm{m}$ is chosen in order to explore the entire range of the predicted phase matching diameters.

At a diameter of $5 \mu \mathrm{m}$, the idler wavelength has a relatively low output of roughly $-67 \mathrm{dBm}$ and do not vary significantly as the OMF is tapered. However, as the OMF reaches the first phase matching diameter $(2.885 \mu \mathrm{m})$ the signal increases significantly by approximately $27 \mathrm{~dB}$, after which it is reduced again as the tapering process continues. This reduction can be attributed to the reduction in the length of OMF which is at the phase matching diameter. The downward trend continues as the fiber is tapered further until the diameter reaches the second phase matching diameter $(799.5 \mathrm{~nm})$, at which point the idler output increases by more than $15 \mathrm{~dB}$. The idler output then reduces in intensity again as it reaches $0.5 \mu \mathrm{m}$. It can be observed that a maximum enhancement of more than $25 \mathrm{~dB}$ is observed for a $6 \mathrm{~mm}$ long OMF, at a diameter of approximately $0.8 \mu \mathrm{m}$.

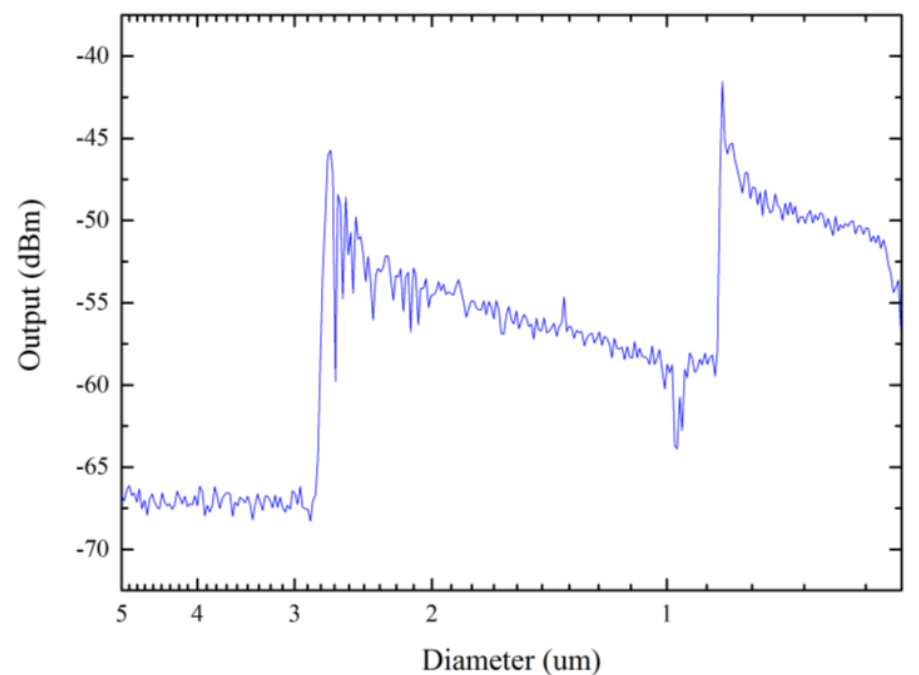

Figure 4: Evolution of the output of the FWM idler signal with OMF diameter from $5 \mu \mathrm{m}$ to $0.5 \mu \mathrm{m}$. The enhancement resulting from phase matching can clearly be distinguished, corresponding to the two phase matching diameters as shown in figure 1.

In summary, we have demonstrated the enhancement of FWM signals by using OMF by exploiting their tailorable dispersion characteristics. It is shown that an enhancement of more than $25 \mathrm{~dB}$ is possible with a 6-mmlong OMF. In principle, this technique can be extended to any wavelength, allowing for the generation of almost arbitrary wavelengths, assuming that other nonlinear effects such as self-phase modulation or cross phase modulation do not dominate.

\section{Acknowledgement}

The authors would like to thank O. Tarasenko and W. Margulis from Acreo Fiberlab who manufactured the fiber used for the PPSF and P. Kazansky at the Optoelectronics Research Centre of the University of Southampton for access to the necessary technical facilities.

\section{References}

[1] A. Cerqueira Sodre, J. M. Chavez Boggio, A. A. Rieznik, H. E. Hernandez-Figueroa, H. L. Fragnito, and J. C. Knight. "Highly efficient generation of broadband cascaded four-wave mixing products." Opt. Express 16, 2816-2828 (2008).

[2] R. L. Carman, R. Y. Chiao, and P. L. Kelley. "Observation of degenerate stimulated four-photon interaction and four-wave parametric amplification." Phys. Rev. Lett. 17, 1281 (1966).

[3] R. H. Stolen. "Phase-matched-stimulated four-photon mixing in silica-fiber waveguides." IEEE J. Quantum Electron 11, 100-103 (1975).

[4] K. Inoue. "Four-wave mixing in an optical fiber in the zero-dispersion wavelength region." J. Lightwave Technol. 10, 1553-1561 (1992).

[5] J.E. Sharping, M. Fiorentino, A. Coker, P. Kumar, and R.S. Windeler. "Four-wave mixing in microstructure fiber." Opt. Lett. 26, 1048-1050 (2001).

[6] J. Hansryd, P. Andrekson, M. Westlund, J. Li, and P. Hedekvist. "Fiber-based optical parametric amplifiers and their applications." IEEE J. Sel. Top. Quantum Electron. 8, 506-520 (2002).

[7] G. Brambilla, "Optical fibre nanowires and microwires: a review." J. Opt. 12, 043001 (2010).

[8] Y.H. Li, Y. Y. Zhao, and L. J. Wang. "Demonstration of almost octave-spanning cascaded four-wave mixing in optical microfibers." Opt. Lett. 37, 3441-3443 (2012).

[9] V. Grubsky, and A. Savchenko. "Glass micro-fibers for efficient third harmonic generation." Opt. Express 13, 6798-6806 (2005). 